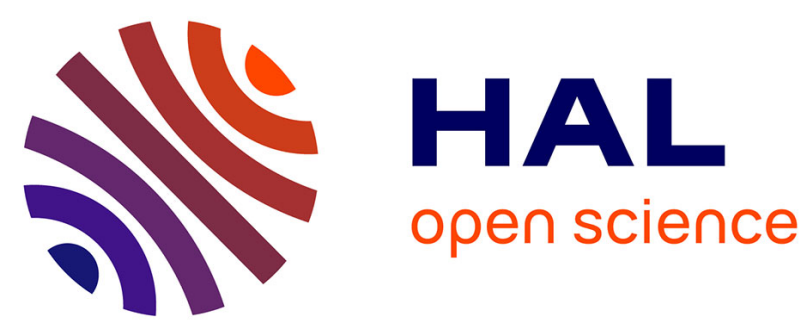

\title{
Single Photon Avalanche Diode with Monte Carlo Simulations: PDE, Jitter and Quench Probability
}

Marco G. Pala, D. Rideau, Y. Oussaiti, J. Grebot, R. Helleboid, A. Lopez, G. Mugny, E. Bourreau, D. Golanski, B. Mamdy, et al.

\section{- To cite this version:}

Marco G. Pala, D. Rideau, Y. Oussaiti, J. Grebot, R. Helleboid, et al.. Single Photon Avalanche Diode with Monte Carlo Simulations: PDE, Jitter and Quench Probability. 2021 International Conference on Simulation of Semiconductor Processes and Devices (SISPAD), Sep 2021, Dallas, United States. pp.293-296, 10.1109/SISPAD54002.2021.9592567 . hal-03445562

\section{HAL Id: hal-03445562 \\ https://hal.science/hal-03445562}

Submitted on 24 Nov 2021

HAL is a multi-disciplinary open access archive for the deposit and dissemination of scientific research documents, whether they are published or not. The documents may come from teaching and research institutions in France or abroad, or from public or private research centers.
L'archive ouverte pluridisciplinaire HAL, est destinée au dépôt et à la diffusion de documents scientifiques de niveau recherche, publiés ou non, émanant des établissements d'enseignement et de recherche français ou étrangers, des laboratoires publics ou privés. 


\section{Single Photon Avalanche Diode with Monte Carlo Simulations: PDE, Jitter and Quench Probability}

\author{
D. Rideau, Y. Oussaiti, J. Grebot, \\ R. Helleboid, A. Lopez*, G. Mugny, \\ E. Bourreau, D. Golanski, B. Mamdy, \\ H. Wehbe Alause, I. Nicholson*, S. Pellegrini*, \\ C.E. Vlimant* and M. Agnew* \\ STMicroelectronics Crolles, France \\ *STMicroelectronics Edinburgh, UK \\ Email: denis.rideau@st.com
}

\author{
T. Cazimajou, M. Pala, J. Saint-Martin \\ and P. Dollfus \\ Centre de Nanosciences et de Nanotechnologies \\ Université Paris-Saclay, Palaiseau, France \\ Email: marco.pala@c2n.upsaclay.fr
}

\section{Introduction}

Single Photon Avalanche Diodes (SPAD) are key optoelectronic detectors for medical imaging, camera ranging and automotive laser imaging detection and ranging (LiDAR) applications. Today, most of SPADs in the Time Of Flight (TOF) market are composed of a micrometric Silicon PN junction associated to a proximity CMOS electronics biasing the system above the breakdown voltage. These devices present low noise, high pixel-matrix integration capabilities, but their Photon Detection Efficiency (PDE) is relatively modest in the near infrared operating region (achieving typically only few percent at a wavelength of $940 \mathrm{~nm}$ ). A considerable optimisation of the SPAD design is currently on going at an industrial level with a view of increasing the PDE without compromising the timing statistic response to avalanche (Jitter) and its quench probability. Within these perspectives, standard commercial Technology-Computer-AssistedDesign (TCAD) simulations can only provide a limited guidance for technological and design splits optimisation since they are based on deterministic solvers that are unable to capture these stochastic figures of merit. Since the seminal work from Spinelli [1] clearly showing that Monte Carlo simulations predictions can compare favourably with experimental measurements of PDE and timing resolution, the Monte Carlo method can be considered to be a useful one for the design of improved structures. It has been recently applied to optimize the PDE and Jitter of Silicon [3] and InGaAs SPADs [4], [5]. In this abstract we report a rigorous comparison between Monte Carlo predictions and measurements of PDE and Jitter. We also discuss in detail, the quench probability of these diodes once in avalanche. This latter point has rarely been discussed in literature and to the best of our knowledge never addressed within a Monte Carlo perspective.

\section{Monte Carlo simulation setup}

A successful methodology for Monte Carlo simulation, combines accurate descriptions of the Design Layouts, Process simulations [6], followed by the device simulation itself. In what follows, for PDE and Jitter simulations we used the full band ensemble Monte Carlo solver of Ref [7] within the frozen field approximation, providing an accurate description of the carrier diffusion within the device and the few ps following the onset of the avalanche. The entire transient response of the device, including the quenching of the avalanche, is obtained with a mixedmode analysis coupling self consistently the efficient Effective-Mass-based ensemble Monte Carlo solver [8], with the so called 'passive quench' biasing circuit (Figure 7). Monte Carlo solvers account for impurity scattering, phonon scattering and Impact Ionization (II) and have been calibrated in order to accurately reproduce mobility, and II coefficients measured in bulk silicon [7], [8] ( full band Montre Carlo solver see e.g. Figure 1).

\section{PDE, and Jitter}

Figure 2 shows simulations of the avalanche currents in a typical SPAD device. As can be seen, the avalanche build up shows a clear temporal statistical 

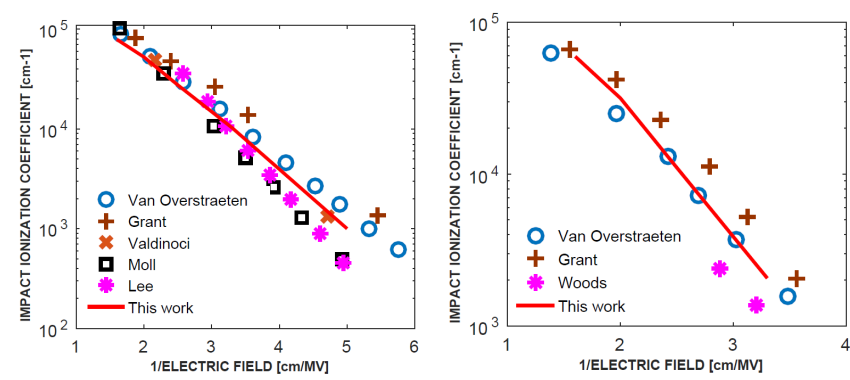

Fig. 1. Simulated II coefficients for electrons (left) and holes (right) compared to measurements in bulk silicon (see [7] for details).

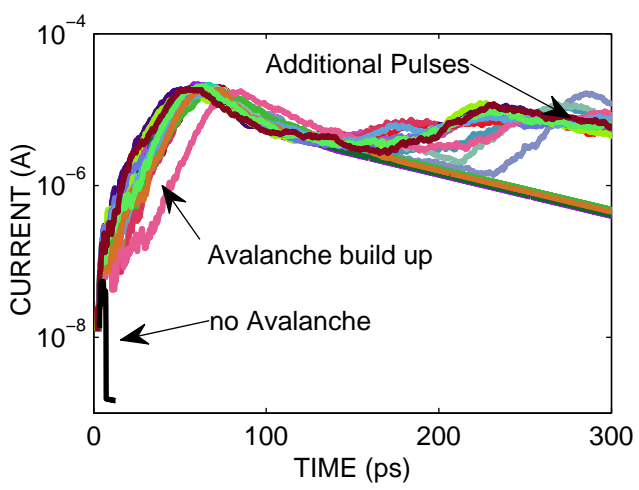

Fig. 2. Statistical Monte Carlo simulation of the current in a SPAD device during the avalanche build up and its quench (corresponding to the SPAD recharge phase for $t>100 \mathrm{ps}$ ).
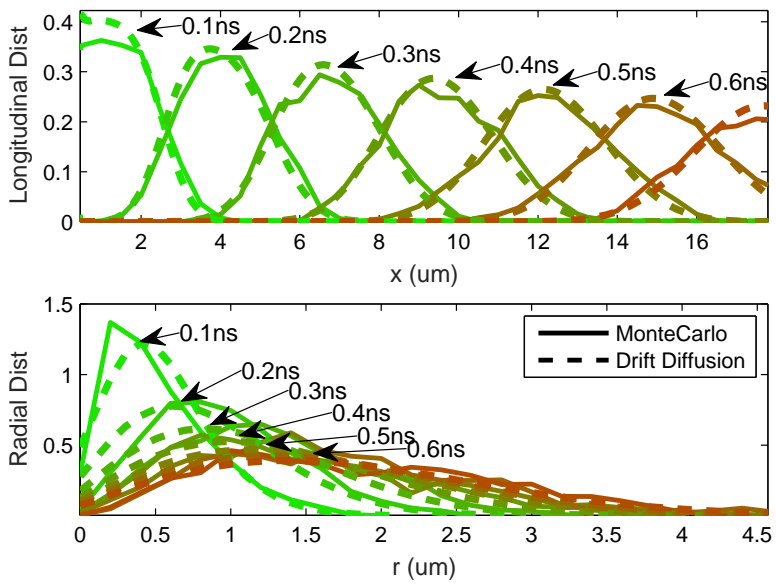

Fig. 3. Solution of the drift and diffusion carriers equation (dashed lines) along (and transvers to) the applied electric field $F_{x}=0.04 \mathrm{MV} / \mathrm{cm}$ compared to the Monte Carlo distributions (lines) for carriers injected at $x=0$ for $t=0$. The field value is lower than the avalanche one of $F_{a v} \approx 0.6 \mathrm{MV} / \mathrm{cm}$ at $T=$ $300 K$.

spread and in certain simulations avanlanche does not occurs. The latter case is related to the PDE

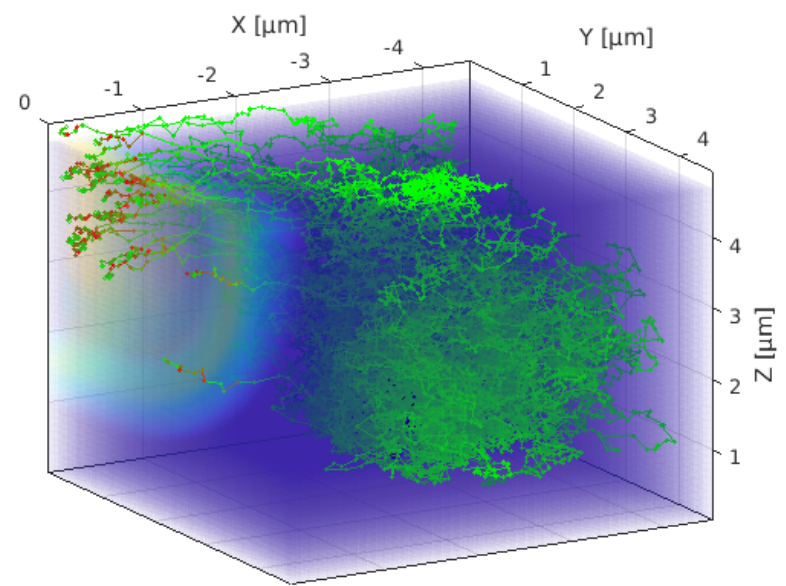

Fig. 4. Photo-generated carrier random trajectories within a SPAD device (1/4 of the structure is shown). The carriers energy depends on the position in the device: low energy values are shown with green segments, higher energy values are shown with red segments. The electric field is shown with transparent colors from bleu (low field) to red (high field); $T=300 K$

measuring the detection efficiency of the SPAD, while the former is related to the timing Jitter. This later is usually defined as Full-Width a Half-Maximum (FWHM) statistical distribution of time to detect avalanche breakdown, but reducing the distribution tail also matters as it can impact the TOF measurement accuracy. Jitter can be inferred from the avalanche build up time itself, but also from the carrier transit time within the device toward the junction [2]. While the build up typically occurs within few tens of ps (see e.g. the spread of the build up current in Figure 2 or the onset of the voltage across the diode in Figure 8), the carrier drift and diffusion can be longer, specially for depleted SPAD [10] (in which a large collection region extend beyond the high field avalanche region). Such random carrier motion is highlighted in Figure 3 in which the electron spacial distribution is calculated by means of Monte Carlo simulations (lines) at various simulation time along (and transfers to) the electric field direction. At relatively low field values $(F<0.1 \mathrm{MV} / \mathrm{cm})$ these distributions can be accurately predicted using the solution of the carrier drift and diffusion equation [2] (dashed lines). However, in a real device, with position-dependent field, the value of which can extend beyond the avalanche one of $F_{a v} \approx 0.6 \mathrm{MV} / \mathrm{cm}$ at $T=300 K$, the situation is more subtle as shown in Figure 4. Several photo-generated carriers random trajectories are shown together with their energy. In the low field regions, a low energy (shown with green 
segments) and a random diffusive motion can be seen, while in the SPAD high field region, the energy rapidly increases (red segments) and the carriers drift along the field lines. This is in this latter region that the avalanche can occur.

Figures 5 and 6 show a comparison between Monte Carlo predictions and measurements. The PDE and the Jitter are obtained from a large (typically 5000) set of simulations using a random position within the device for the initial photo-generated electron-hole pair (we verified [9] that at the studied wavelength of $940 \mathrm{~nm}$ the carrier absorption is nearly uniform along the depth of the studied devices).

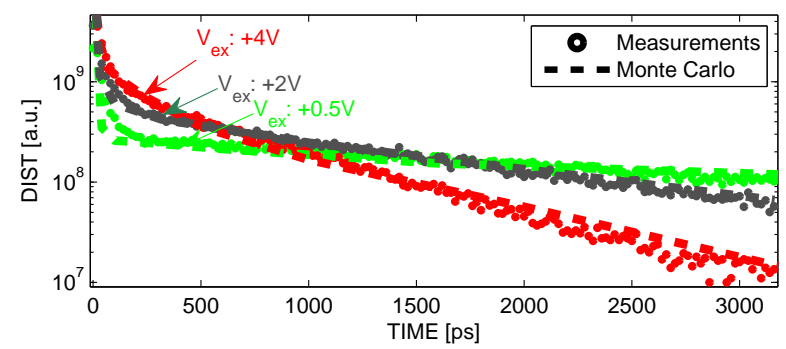

Fig. 5. Measurement and simulation of the Jitter tail due to carrier diffusion within the collection volume of a nonoptimized SPAD. In that case, and for the lower excess voltage $V_{e x}$ a long carrier transit time toward the junction can occur, giving rise to a clear increase of the jitter tail.

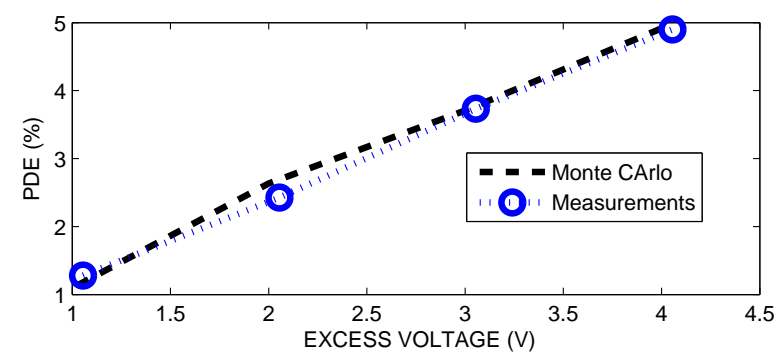

Fig. 6. Simulation of PDE as a function of applied excess voltage $\left(V_{H}=B_{V}+V_{e x}\right)$ compared to measurements.

\section{Statistics of the Quench}

Figure 2 also shows that in certain cases, the current does not vanish but increases again giving rise to additional pulses. This is a statistical phenomenon that is governed by the carrier evacuation time, but also by the quench circuit and its recharge time. The circuit is shown in Figure 7 together with the measured Pulse Width (PW) distribution after the inverter stage. Applying various biases on the Anode transistor modifies the equivalent quench resistor (in series with the SPAD device) and drastically degrades the PW distribution. This contrasts with the usual SPAD trends where the pulse width decreases as the quenching resistor decreases because of the shorter recharge time. This is exemplified in small diameter SPADs exhibiting small diode capacitance. We performed a mixed-mode EMA-based 3D Monte Carlo analysis of a small SPAD PN junction with a realistic doping profile obtained from process simulation [6] (with length $\mathrm{L}=1300 \mathrm{~nm}$ and square cross section of width $\mathrm{W}=300 \mathrm{~nm})$ connected in series with a passive $R C$-quenching circuit (mimicking the quenching circuit shown in Figure 7). The equivalent $R C$-time constant of the moving node is lower than $100 \mathrm{ps}$ : for low resistor values, the moving-Anode of the SPAD can be rearmed at $V_{H}$ faster than the carrier evacuation time. This gives rise to an effective increased PW because an additional avalanche (or several avalanches - see Figure 8) occurs before the anode cross the inverter threshold. Monte Carlo simulations of the SPAD voltage $\left(V_{D}=V_{K}-V_{A}\right)$ are shown in Figure 8 and the quench probability is calculated from the statistical average of the ratio of simulations in which the SPAD is fully rearmed after $250 \mathrm{ps}$ with the simulations in which the voltage is still below the applied $V_{H}$ voltage. This quench probability $P_{Q}$ increases with the effective quench resistor $R_{Q}$, which is well captured by Monte Carlo simulations as shown in Figure 9. Remarkably, this probability changes from $0 \%$ for $R_{Q}=300 \mathrm{k} \Omega$ to $100 \%$ for $R_{Q}=1.3 \mathrm{M} \Omega$.

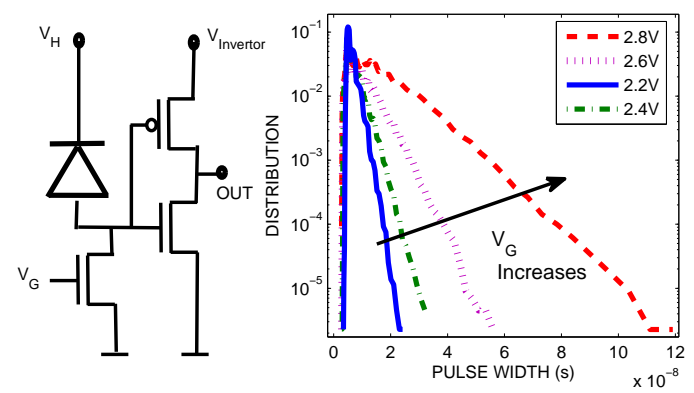

Fig. 7. Passive Quench circuit (left) and measured Pulse Width distribution at the OUT terminal for various applied biases $V_{G}$.

Relevant statistical mixed-mode Monte Carlo analysis require typically hundreds of simulations. Extensive studies in larger and more realistic SPAD 
diodes, the diameter of which can be several tens of microns (and the capacitance several tens of $\mathrm{fF}$ ), would require a drastic increase of the CPU simulation time. Instead, we used an empirical version of the Monte Carlo code, in which the carrier motion is randomly assessed according to the distribution probability shown in Figure 3, and the impact ionization probability computed using ionization integrals along the random carrier trajectory. Such efficient empirical statistical methods make possible detailed analysis of the Quench probability dependence on the SPAD architecture and design at an industrial level. More details on empirical Monte Carlo and its calibration using full band Monte Carlo simulation results will be presented at the conference.

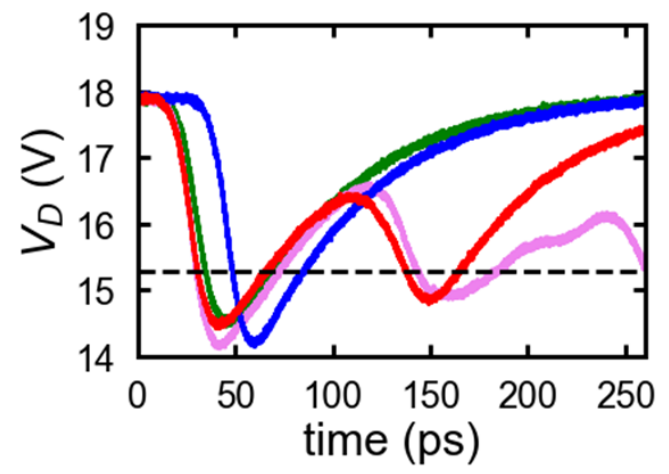

Fig. 8. Monte Carlo simulations of the SPAD biasing with a passive quench resistor of $R_{Q}=700 \mathrm{k} \Omega$. Equivalent SPAD capacitance of $C_{S P A D}=20.7 a F$ and external quench capacitance of $C_{Q}=0.1 f F$. Dashed line represents the Breakdown voltage $B_{V}$.

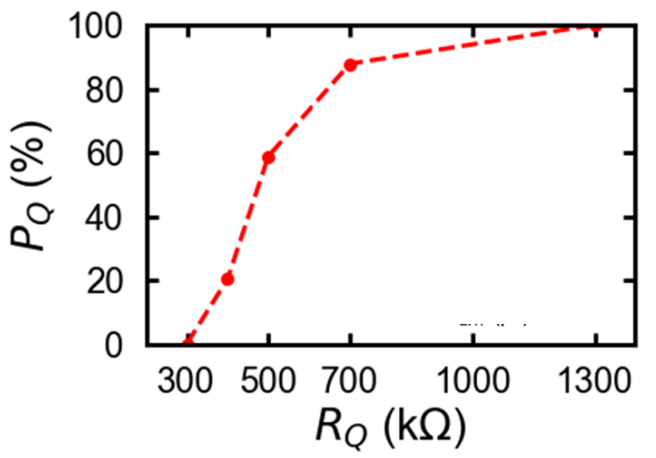

Fig. 9. Monte Carlo simulation of the quench probability $P_{Q}$ as a function of the equivalent quench resistor $R_{Q}$ (see text for details).

\section{References}

[1] A. Spinelli and A. L. Lacaita, "Physics and numerical simulation of single photon avalanche diodes," IEEE Trans. Electron Devices, vol. 44, no. 11, pp. 1931-1943, 1997

[2] C. Jacoboni, P. Lugli, "Monte Carlo Method for Semiconductor Device Simulation", Springer Vienna, ISBN-10: 3211821104, 1989

[3] J. D Petticrew, S. J Dimler, X. Zhou, A. P Morrison, C. H. Tan, J. S. Ng, " Avalanche Breakdown Timing Statistics for Silicon Single Photon Avalanche Diodes", IEEE Journal of Selected Topics in Quantum Electronics, 24(2), 1-6, 2018

[4] D. Dolgos, H. Meier, A. Schenk and B. Witzigmann, "Fullband Monte Carlo simulation of single photon avalanche diodes," 2013 IEEE Photonics Conference, Bellevue, WA, pp. 360-361, 2013

[5] D. Dolgos, H. Meier, A. Schenk and B. Witzigmann"Fullband Monte Carlo simulation of high-energy carrier transport in single photon avalanche diodes with multiplication layers made of InPInAlAsand GaAs" Journal of Applied Physics, vol. 111, no. 10, p. 104508, 2012

[6] Present process simulations have been performed using Sentaurus SProcess, https://www.synopsys.com/silicon/tcad/processsimulation/sentaurus-process.html

[7] M Michaillat, D. Rideau, F. Aniel , C. Tavernier and H. Jaouen, "Monte Carlo simulation of high-energy transport of electrons and holes in bulk SiGeC alloys", J. Phys.: Conf. Ser. 193 012037, 2009

[8] P. Dollfus, A. Bournel, S. Galdin-Retailleau, S. Barraud, and P. Hesto, "Effect of discrete impurities on electron transport in ultra-short MOSFET using 3D Monte Carlo simulation", IEEE Trans. Electron Devices, vol. 51 (5), 749-756, 2004

[9] For light absorption we used Lumerical (simulations results not shown here)

[10] M. Hofbauer, B. Steindl, and H. Zimmermann, "Temperature Dependence of Dark Count Rate and After Pulsing of a Single-Photon Avalanche Diode with an Integrated Active Quenching Circuit in $0.35 \mu \mathrm{m}$ CMOS",Journal of Sensors, Vol 2018, Article ID 9585931, 7, 2018 